\title{
Attachment of the N-Terminal Domain of Salmonella typhimurium AhpF to Escherichia coli Thioredoxin Reductase Confers AhpC Reductase Activity but Does Not Affect Thioredoxin Reductase Activity ${ }^{\dagger}$
}

\author{
C. Michael Reynolds and Leslie B. Poole* \\ Department of Biochemistry, Wake Forest University School of Medicine, Winston-Salem, North Carolina 27157
}

Received April 12, 2000

\begin{abstract}
AhpF of Salmonella typhimurium, the flavoprotein reductase required for catalytic turnover of $\mathrm{AhpC}$ with hydroperoxide substrates in the alkyl hydroperoxide reductase system, is a $57 \mathrm{kDa}$ protein with homology to thioredoxin reductase (TrR) from Escherichia coli. Like TrR, AhpF employs tightly bound FAD and redox-active disulfide center(s) in catalyzing electron transfer from reduced pyridine nucleotides to the disulfide bond of its protein substrate. Homology of AhpF to the smaller $(35 \mathrm{kDa}) \operatorname{TrR}$ protein occurs in the C-terminal part of AhpF; a stretch of about 200 amino acids at the N-terminus of $\mathrm{AhpF}$ contains an additional redox-active disulfide center and is required for catalysis of AhpC reduction. We have demonstrated that fusion of the N-terminal 207 amino acids of AhpF to full-length TrR results in a chimeric protein $(\mathrm{Nt}-\mathrm{TrR})$ with essentially the same catalytic efficiency $\left(k_{\mathrm{cat}} / K_{\mathrm{m}}\right)$ as $\mathrm{AhpF}$ in AhpC reductase assays; both $k_{\text {cat }}$ and the $K_{\mathrm{m}}$ for AhpC are decreased about 3-4-fold for Nt-TrR compared with AhpF. In addition, Nt-TrR retains essentially full TrR activity. Based on results from two mutants of Nt-TrR (C129,132S and C342,345S), AhpC reductase activity requires both centers while TrR activity requires only the C-terminal-most disulfide center in Nt-TrR. The high catalytic efficiency with which $\mathrm{Nt}-\mathrm{TrR}$ can reduce thioredoxin implies that the attached $\mathrm{N}$-terminal domain does not block access of thioredoxin to the TrR-derived Cys342-Cys345 center of Nt-TrR nor does it impede the putative conformational changes that this part of Nt-TrR is proposed to undergo during catalysis. These studies indicate that the $\mathrm{C}$-terminal part of $\mathrm{AhpF}$ and bacterial $\mathrm{TrR}$ have very similar mechanistic properties. These findings also confirm that the $\mathrm{N}$-terminal domain of $\mathrm{AhpF}$ plays a direct role in AhpC reduction.
\end{abstract}

The alkyl hydroperoxide reductase system from Salmonella typhimurium consists of two proteins, $\mathrm{AhpF}$ and $\mathrm{AhpC}$, which catalyze the reduction of organic hydroperoxides to their corresponding alcohols $(1,2) . \mathrm{AhpF}$ is a $57 \mathrm{kDa}$ dimeric flavoprotein that utilizes reducing equivalents from NAD(P)H to reduce its substrate protein, AhpC (a member of the peroxiredoxin family). Reduced $\mathrm{AhpC}$ is a direct reductant of organic hydroperoxides and hydrogen peroxide $(3,4)$. This activity protects cells against oxidative damage to DNA and other cellular macromolecules $(1,5)$.

$\mathrm{AhpF}$ is an FAD-containing pyridine nucleotide:disulfide oxidoreductase related to Escherichia coli thioredoxin reductase $(\mathrm{TrR}) ;^{1}$ residues $208-521$ of $\mathrm{AhpF}$ exhibit $\sim 35 \%$

$\uparrow$ This research was supported by NIH Grant GM-50389 and by Council for Tobacco Research Awards SA006 and 4501 to L.B.P.

* To whom correspondence should be addressed at the Department of Biochemistry, Wake Forest University School of Medicine, Medical Center Blvd., Winston-Salem, NC 27157. Tel.: 336-716-6711; Fax: 336-716-7671; e-mail: lbpoole@wfubmc.edu; URL: http://www.wfubmc.edu/biochem/faculty/Poole/poole.html.

${ }^{1}$ Abbreviations: TrR, thioredoxin reductase; Tr, thioredoxin; $\mathrm{F}[1-$ 202], truncated AhpF including residues 1 through 202; F[208-521], truncated AhpF including methionine plus residues 208 through 521; $\operatorname{tr} x A$, structural gene encoding $\operatorname{Tr} ; \operatorname{tr} x B$, structural gene encoding TrR; $n t$-trxB, chimeric DNA construct encoding residues 1-207 of AhpF followed by $\operatorname{tr} x B$ (starting with codon 2); Nt-TrR, chimeric protein expressed from $n t$-trxB; DTNB, 5, $5^{\prime}$-dithiobis(2-nitrobenzoate); TNB, 2-nitro-5-thiobenzoate; LB, Luria-Bertani medium; SDS, sodium dodecyl sulfate; PCR, polymerase chain reaction; IPTG, isopropyl $\beta$-Dthiogalactopyranoside; Fd/FNR, ferredoxin/ferredoxin-NADP ${ }^{+}$reductase fusion protein; $\mathrm{Fd}$, ferredoxin; FNR, ferredoxin- $\mathrm{NADP}^{+}$reductase. identity to full-length TrR (Figure 1A) $(6,7)$. The additional stretch of about 200 amino acids at the N-terminus of AhpF contains an extra redox-active disulfide center required for catalysis of AhpC reduction (8). Our working hypothesis has been that the $\mathrm{C}$-terminal part of $\mathrm{AhpF}$ undergoes electron transfer from NADH to the TrR-like Cys345-Cys348 redox center via the flavin in a manner quite analogous to those transfers in TrR (Figure 1B,C). The Cys129-Cys132 redox center in the $\mathrm{N}$-terminal domain of $\mathrm{AhpF}$ then acts as a shuttle to bring electrons from the dithiol form of Cys345Cys348 to the active site intersubunit disulfide bond of AhpC (Figure 1C). Data supporting this hypothesis include the lack of AhpC reductase activity for three proteins that have been investigated: (i) the $\mathrm{C} 129,132 \mathrm{~S}$ mutant of $\mathrm{AhpF}(8)$; (ii) the truncated AhpF protein lacking 207 amino acids at the N-terminus (9); (iii) and TrR when tested in the absence of thioredoxin $(\mathrm{Tr})(3)$.

Other experiments have also given a clear indication of the direct role the N-terminal domain of AhpF plays in AhpC reduction. When tested for AhpC reductase activity using a fluorophore-labeled AhpC (the C46S mutant labeled at Cys165) (4, 10), only the independently expressed, reduced $\mathrm{N}$-terminal domain of $\mathrm{AhpF}$ or intact $\mathrm{AhpF}$ were effective reductants of this substrate; the complementary $\mathrm{C}$-terminal fragment of $\mathrm{AhpF}$ was essentially devoid of $\mathrm{AhpC}$ reductase activity (9). In separate experiments, disulfide-linked complexes between $\mathrm{AhpF}$ and $\mathrm{AhpC}$, expected to mimic catalytic intermediates, were engineered between single cysteine 
A

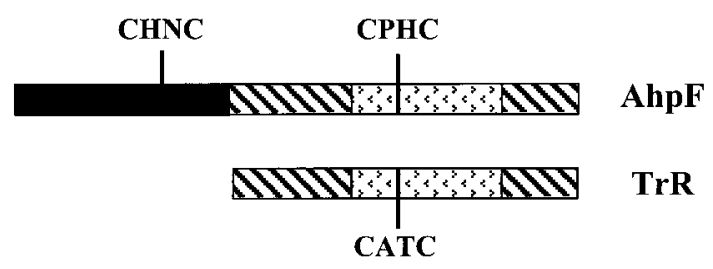

B

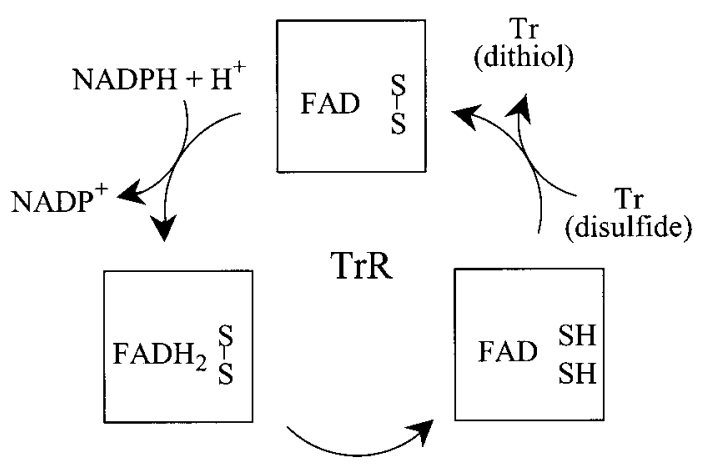

$\mathrm{C}$

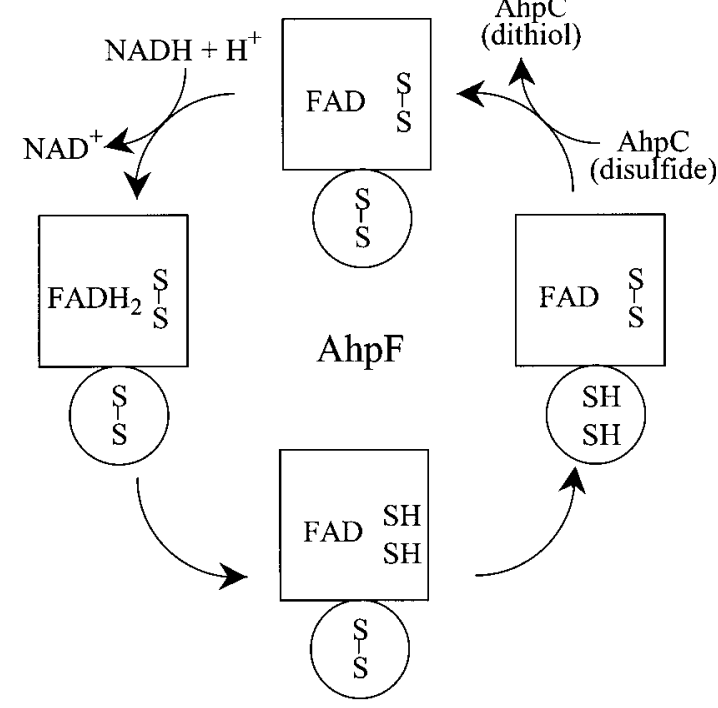

FIGURE 1: Redox centers of $\mathrm{AhpF}$ and thioredoxin reductase (TrR): primary structures and sequence of electron transfers. Shown in panel A are the noncontiguous parts of the flavin binding domains (hatched), the pyridine nucleotide binding domains (stippled) containing a conserved redox-active disulfide center (CXXC), and the N-terminal domain of AhpF (black) with an additional redoxactive disulfide center (CQNC). During the catalytic cycle of TrR (panel B), two electrons from NADPH (accompanied by two $\mathrm{H}^{+}$) are transferred to the flavin, then to the redox-active disulfide center, and finally to the thioredoxin (Tr) substrate. The catalytic cycle of $\mathrm{AhpF}$ (panel C) proceeds via the analogous electron transfers from $\mathrm{NADH}$ to flavin to the C-terminal-most disulfide center, then continues with electron transfers to the N-terminal-most disulfide center (with the $\mathrm{N}$-terminal domain represented by the circle) and to the intersubunit disulfide bond in the active site of the AhpC substrate.

mutants of the two proteins (10). Such complexes were only possible to generate, however, if linkage of $\mathrm{AhpC}$ to $\mathrm{AhpF}$ occurred through one of the two $\mathrm{N}$-terminal cysteine residues, but not through either of the two C-terminal active site cysteine residues, of AhpF. As indicated above, E. coli $\mathrm{TrR}$ is also capable of AhpC reduction, but only if $\mathrm{Tr}$ is present to mediate this electron transfer. These data all suggest that the N-terminal domain of AhpF can be likened to an attached Tr-like redox carrier playing an intermediary role in electron transfer between the C-terminal part of AhpF and AhpC (10). Recent recognition that the $\mathrm{N}$-terminus of AhpF contains a tandem repeat of Tr-like folds with the redox-active disulfide residing in the second of the two folds has been an interesting source of additional support for this hypothesis (9).

$\operatorname{TrR}$, the flavin-containing pyridine nucleotide:disulfide oxidoreductase most closely related to AhpF, plays a critical role in the maintenance of cell redox status through regeneration of the pool of reduced $\mathrm{Tr}$ using electrons from NADPH (11). Reduced Tr has been implicated in a variety of physiological functions, including the reduction of disulfide bonds in enzymes such as ribonucleotide reductase which require such a step for turnover $(12,13)$. It has recently been recognized that $\operatorname{TrR}$ in many eukaryotic organisms is structurally quite distinct from bacterial $\operatorname{TrR}$ and is likely to catalyze Tr reduction through an additional redox center at the C-terminus of the protein $(14-16)$. This protein is therefore larger $(\sim 55 \mathrm{kDa})$ than its bacterial orthologue and is, in fact, more closely related to the group of pyridine nucleotide:disulfide oxidoreductases which includes glutathione reductase and lipoamide dehydrogenase. Bacterial $\mathrm{TrR}$, in contrast, is smaller $(\sim 35 \mathrm{kDa})$ and functions through one tightly bound FAD and one redox-active disulfide center per subunit (Figure 1B), but appears to rely on conformational changes during catalysis to shuttle electrons to $\operatorname{Tr}(11$, 16, 17). It is notable that, while $\mathrm{AhpF}$ is structurally more closely related to bacterial $\operatorname{TrR}$ (Figure 1A), its catalytic mechanism, which employs an additional redox center in another part of the protein (Figure 1C), has similarities to that of the high $M_{\mathrm{r}} \operatorname{TrR}$. Both classes of $\operatorname{TrR}$ are similar to $\mathrm{AhpF}$ in catalyzing the pyridine nucleotide-dependent reduction of a disulfide-containing protein substrate. Interestingly, though, the three proteins (high and low $M_{\mathrm{r}} \mathrm{TrR}$ and $\mathrm{AhpF}$ ) appear to accomplish their tasks through distinct pathways that include some combination of conformational changes and additional redox centers $(16,18)$.

Given these similarities and distinctions, we set out to test the possibility that a chimeric protein with high AhpC reductase activity could be constructed through appendage of the N-terminal domain of AhpF to E. coli TrR. As described herein, our chimeric construct not only has high AhpC reductase activity but also has essentially full TrR activity. This striking result is discussed in terms of its implications for the structures and mechanisms of both parent proteins, AhpF and bacterial $\operatorname{TrR}$.

\section{EXPERIMENTAL PROCEDURES}

Materials. NADPH and NADH were purchased from Boehringer Mannheim. Sigma was the supplier of FAD, cumene hydroperoxide $(80 \%)$, molecular biology grade ammonium sulfate, methyl viologen, bovine pancreas insulin, streptomycin sulfate, protocatechuic acid, and protocatechuate 3,4-dioxygenase. Difco bacteriological media, sodium dithionite, and organic solvents were from Fisher. Other reagents and buffer components including DTNB and SDS were purchased from Gibco BRL. Restriction enzymes, DNA-modifying enzymes, and DNA polymerases were obtained from Promega or New England Biolabs. The standard buffer used was $25 \mathrm{mM}$ potassium phosphate at pH 7.0, with $1 \mathrm{mM}$ EDTA, unless otherwise indicated. 


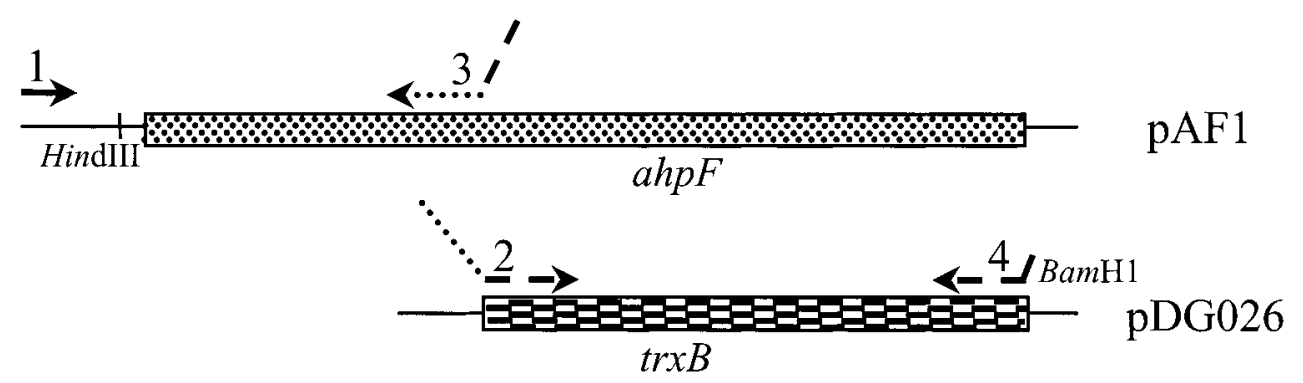

FIGURE 2: Construction of the expression plasmid for Nt-TrR using the megaprimer PCR method. Reactions with the primers and the wild-type $a h p F$ and $\operatorname{tr} x B$ genes as templates were carried out as described under Experimental Procedures.

Molecular Biology and Protein Purification Methods. Megaprimer polymerase chain reaction (PCR) (19) was used to join the N-terminal portion of $S$. typhimurium ahpF (codons 1-207) to full-length $E$. coli trx $B$ to create $n t-\operatorname{tr} x B$, the DNA construct engineered to encode the chimeric protein Nt-TrR. ${ }^{2}$ Four primers were synthesized to allow for the joining of the two gene fragments and construction of the expression plasmid as shown in Figure 2 (all oligonucleotides were synthesized in the DNA Synthesis Core Laboratory of the Comprehensive Cancer Center of Wake Forest University). pAF1 containing $\operatorname{ahpF}(2)$ and pDG026 containing trxB (20) were used as templates in megaprimer PCR to construct $n t$-trx $B$ as follows. Primers 2 and 3 (5'-CGCGCGGCGGAAGCGCTGGGCACGACCAAACACAG-3' and 5'-CTGTGTTTGGTCGTGCCCAGCGCTTCCGCCGCGCG-3', respectively) are complementary oligonucleotides which contain sequence prior to and including the codon for residue 207 (shown in boldface) of $a h p F$, followed by the first 20 bases, minus the ATG start codon, of the TrR-coding $\operatorname{tr} x B$ gene. The first PCR reaction utilized Primer 1 (5'-TGCAGGTTAACCTGGC- $3^{\prime}$ ) in 100-fold excess over Primer 3 to amplify the N-terminal sequence of $a h p F$. The second PCR reaction utilized a 100-fold excess of Primer 4 (5'-CCGGATCCTTATTTTGCGTCAGCTAAACC-3') over Primer 2 to amplify $\operatorname{tr} x B$. The products of the two reactions, the megaprimers, were mixed, allowed to anneal, and then extended. Primers 1 and 4 were then used to amplify the chimeric construct. PCR conditions were $95^{\circ} \mathrm{C}$ for $30 \mathrm{~s}, 55$ ${ }^{\circ} \mathrm{C}$ for $45 \mathrm{~s}$, and $72{ }^{\circ} \mathrm{C}$ for $1.5 \mathrm{~min}$, for 35 cycles. Extension and amplification of the megaprimers were done at the same temperatures for 20 cycles and 35 cycles, respectively. A HindIII site present upstream of $a h p F$ in pAF1 and the engineered BamHI site (italics) of Primer 4 were used for subcloning of the PCR product into the corresponding sites of pOXO4 (21).

In addition to $n t-\operatorname{trx} B$, a second construct was made using this protocol, but with a pAF1 plasmid encoding a $\mathrm{C} 129,132 \mathrm{~S}$ mutation in $\operatorname{ahpF}(8)$ as the template in the first PCR reaction. Both chimeric genes were sequenced in their entirety using the DNA sequence analysis core laboratory of the Comprehensive Cancer Center of Wake Forest University.

A third chimeric construct was created by mutagenesis of "wild-type" $n t$-trxB using the GeneEditor in vitro SiteDirected Mutagenesis System from Promega. The mutagenic

\footnotetext{
${ }^{2} \mathrm{Nt}-\mathrm{TrR}$ was designed on the basis of sequence alignment results between $\mathrm{AhpF}$ and $\mathrm{TrR}$, where the region in common begins with residue 208 of $\mathrm{AhpF}$ and residue 1 of TrR (Gly1 of TrR is encoded by the second codon of $\operatorname{tr} x B$ ).
}

oligonucleotide, 5'-GGGTTTCTGCTAGCGCAACCTCCGACGGTTTC-3' (with the sites of the new codons underlined and an engineered NheI site in italics), was used to replace both Cys342 and Cys345 (formerly Cys135 and Cys 138 of TrR, respectively) with serine residues. Following mutagenesis of the PCR 2.1 vector (Invitrogen) into which $n t$-trx $B$ had been ligated, mutants were screened by digestion with NheI. The mutated construct was then subcloned into pOXO4 as described in Figure 2 and verified by sequence analysis of the entire gene.

Cultures of E. coli strain JM109 (DE3) harboring each of the expression plasmids were grown in $10 \mathrm{~L}$ of chloramphenicol-containing Luria-Bertani (LB) medium supplemented with $0.2 \%$ glucose in a New Brunswick BioFlo 2000 fermentor after inoculation to $2 \%$ from an overnight culture. Isopropyl $\beta$-D-thiogalactopyranoside (IPTG; $0.4 \mathrm{mM}$ ) was added at $A_{600}=1.4$, and the growth was continued for $3 \mathrm{~h}$. Harvested cell pellets were stored at $-20{ }^{\circ} \mathrm{C}$ until needed. For purification of Nt-TrR proteins, streptomycin sulfatetreated cell extracts from mechanical cell disruption were brought to $30 \%$ ammonium sulfate in the standard buffer, centrifuged to remove the pellet, and then brought to $70 \%$ ammonium sulfate and repelleted essentially as described previously (2). The resuspended pellet dialyzed against standard buffer was applied to a preequilibrated DEAEcellulose column (Whatman DE52), washed with $80 \mathrm{mM}$ potassium phosphate buffer ( $\mathrm{pH}$ 7.0, including $1 \mathrm{mM}$ EDTA), and then eluted with a linear gradient of potassium phosphate buffer from 80 to $160 \mathrm{mM}$. Fractions containing the protein of interest were analyzed by SDS-polyacrylamide gel electrophoresis and $A_{280} / A_{450}$ ratios, pooled, and loaded directly onto an Affi-Gel Blue (Bio-Rad) column preequilibrated in standard buffer. The column was washed, and then eluted with a linear gradient from standard buffer to $0.6 \mathrm{M}$ potassium phosphate, $\mathrm{pH} 7.0$, containing $0.3 \mathrm{M} \mathrm{NaCl}$ and 1 $\mathrm{mM}$ EDTA. Pooled fractions were brought to $20 \%$ ammonium sulfate and applied to a Phenyl Sepharose fast flow column preequilibrated with standard buffer containing $20 \%$ ammonium sulfate. The column was washed, and then eluted with a linear gradient of the standard buffer containing from $20 \%$ to $0 \%$ ammonium sulfate. Pure proteins were pooled, dialyzed against standard buffer, and stored at $-20^{\circ} \mathrm{C}$ until further use. Ten liters of bacterial culture yielded about 90 $\mathrm{mg}$ of pure Nt-TrR proteins.

A DNA fragment containing the gene coding for thioredoxin, $\operatorname{trx} A$, was amplified from $E$. coli genomic DNA by PCR using the following oligonucleotide primers: Primer 5 (N-terminal primer), 5'-CCGAAGCTTAGGAGGAAGTAT-

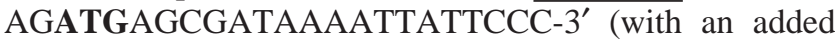


HindIII site in italics, an engineered ribosome binding site underlined, and the ATG start codon in boldface); and Primer 6 (C-terminal primer), 5'-CCGGATCCTTACGCCAGGTTAGCGTC-3' (with an added BamHI site in italics). Genomic DNA was prepared from an overnight culture of an E. coli K-12 strain (XL1-Blue) (22) and used as a template for PCR as described for the generation of $n t-\operatorname{tr} x B$. The PCR product was ligated into the Invitrogen PCR 2.1 TA cloning vector, the resulting plasmid was digested with HindIII and $B a m \mathrm{HI}$, and the $\operatorname{tr} x A$-containing fragment was subcloned into the corresponding sites of pOXO4 to create pTrxA and verified by sequence analysis.

Thioredoxin was expressed and purified from $8 \mathrm{~L}$ of IPTGinduced E. coli JM109 (DE3) cell harboring pTrxA by a slight modification of methods previously established (23) (a Whatman DE52 column was used instead of the Q Sepharose Fast Flow column, and a BioGel A-0.5m column was employed for the last step of the purification instead of Sephadex G-50). Fractions containing pure $\mathrm{Tr}$ as analyzed by SDS-polyacrylamide gel electrophoresis were pooled, dialyzed against standard buffer, and stored at $-20{ }^{\circ} \mathrm{C}$ until further use.

E. coli TrR was cloned, expressed, and purified as described previously (9), as were $S$. typhimurium AhpF and AhpC (2).

Spectroscopic Experiments. A thermostated Milton Roy Spectronic 3000 diode array spectrophotometer with 0.35 $\mathrm{nm}$ resolution was used to collect all absorbance spectra and to carry out enzymatic assay and anaerobic titration experiments. Fluorescence spectra were recorded with an SLM Aminco Bowman Series 2 luminescence spectrophotometer. Assays were also done using a thermostated Applied Photophysics DX.17MV stopped-flow spectrofluorometer. Spectrophotometric titrations were conducted using a glass titration cuvette as described previously (3). All reductive titrations were carried out in the dark and used protein preparations lacking EDTA (removed by extensive washing in Centricon CM-50 ultrafiltration units) to avoid light- and/ or EDTA-catalyzed production of flavin semiquinone by the partially reduced protein. Dithionite and NADPH titrating solutions were prepared in buffer (50 mM Tris- $\mathrm{HCl}, \mathrm{pH} 8.0)$ bubbled for 30 min with oxygen-free argon, loaded into the titrating syringe, and standardized prior to each experiment. The dithionite solution was standardized by anaerobic titration of a solution of free FAD. Methyl viologen was added at a molar ratio of $1: 100$ relative to $\mathrm{Nt}-\mathrm{TrR}$ (or at 1:10 for the mutants of Nt-TrR, which were slower to equilibrate) to promote equilibration between redox centers during dithionite titrations. Spectral data were not corrected for dilution which was less than 5\% overall. All anaerobic experiments included an oxygen-scrubbing system consisting of protocatechuic acid and protocatechuate 3,4-dioxygenase.

Extinction coefficients used for all reduced and oxidized pyridine nucleotides, free FAD, and TNB were those previously reported (2). Microbiuret assays to determine protein content and analyses of flavin content were carried out as previously described $(2,3)$.

Determination of Redox Potentials. Tr or F[1-202] (20 $\mu \mathrm{M}$ ) was mixed with $50 \mu \mathrm{M}$ NADPH in a total volume of $500 \mu \mathrm{L}$ in the standard buffer at $25^{\circ} \mathrm{C}$ followed by addition of $10 \mathrm{nM}$ TrR, and then excess $\mathrm{NADP}^{+}$(to final concentrations between 200 and $600 \mu \mathrm{M}$ ) as was described previously
(24). From the equilibrium concentrations of oxidized and reduced proteins and pyridine nucleotides, midpoint reduction potentials were calculated according to the Nernst equation:

$$
\begin{aligned}
& E_{\mathrm{o}}{ }^{\prime}(\text { substrate })=E_{\mathrm{o}}{ }^{\prime}\left(\mathrm{NADP}^{+}\right)+ \\
& (R T / n F) \ln \left(\left[\mathrm{NADP}^{+}\right]\left[\text {substrate }_{\text {red }}\right] /[\mathrm{NADPH}]\left[\text { substrate }_{\mathrm{ox}}\right]\right)
\end{aligned}
$$

A value of $-0.315 \mathrm{~V}$ was used as the redox potential of $\mathrm{NADP}^{+}$(25).

Analytical Ultracentrifugation Studies. Sedimentation equilibrium analyses were performed essentially as described previously (9). The concentration of Nt-TrR was varied from 3 to $75 \mu \mathrm{M}$, with $110 \mu \mathrm{L}$ of each sample loaded into three of the six sectors of each cell and buffer in the reference sectors. Global analysis of multiple data sets (both 280 and $454 \mathrm{~nm}$ data) equilibrated at 7300, 8700, 10 400, 12300 , and $14300 \mathrm{rpm}$ was performed using the Windows version of NONLIN (26). Only data of less than 1.0 absorbance unit were used in the analysis. A value of $0.7366 \mathrm{~cm}^{3} \mathrm{~g}^{-1}$ was calculated for the partial specific volume of Nt-TrR from the amino acid composition (27).

Activity Assays and Thiol Quantitations. Standard assays for DTNB reductase activity were carried out essentially as described previously (2), except the assay buffer did not contain ammonium sulfate. Also, NADPH was substituted for NADH in the assay buffer for all proteins except AhpF.

Steady-state peroxidase assays with $\mathrm{AhpC}$ were carried out on the stopped-flow spectrofluorometer at $25{ }^{\circ} \mathrm{C}$ using $0.2 \mu \mathrm{M}$ Nt-TrR or $0.1 \mu \mathrm{M}$ AhpF in the presence of $150 \mu \mathrm{M}$ $\mathrm{NAD}(\mathrm{P}) \mathrm{H}, 1 \mathrm{mM}$ cumene hydroperoxide, $50 \mathrm{mM}$ potassium phosphate at pH 7.0, 0.5 mM EDTA, $100 \mathrm{mM}$ ammonium sulfate, and 1-55 $\mu \mathrm{M}$ AhpC. TrR, Nt-TrR C129,132S and Nt-TrR C342,345S, at 2.5 and $5 \mu \mathrm{M}$ each, were assayed with $14 \mu \mathrm{M} \mathrm{AhpC}$ and found to have minimal activity.

Assays for TrR activity were carried out at $25{ }^{\circ} \mathrm{C}$ using $0.5 \mathrm{~mL}$ of a solution containing $150 \mu \mathrm{M}$ NAD(P)H, $100 \mu \mathrm{M}$ bovine pancreas insulin, $50 \mathrm{mM}$ potassium phosphate at $\mathrm{pH}$ 7.0, and $1 \mathrm{mM}$ EDTA following the method of Holmgren (28). The concentration of $\operatorname{Tr}$ was varied from 0.1 to $32 \mu \mathrm{M}$ with the flavoprotein at $20 \mathrm{nM}$. Complete sets of steadystate assays were not done with AhpF or Nt-TrR C342,345S due to their lack of activity.

Assays for free thiol groups were carried out by incubation of oxidized or NAD(P)H-reduced (anaerobic) protein solutions with guanidine hydrochloride at a final concentration of 4 or $6 \mathrm{M}$, followed by addition of $100 \mu \mathrm{M}$ DTNB.

\section{RESULTS AND DISCUSSION}

The N-Terminus of AhpF, F[1-202], as a Substrate for TrR; Catalytic Properties and Redox Potentials. The Nterminus of AhpF was previously shown to be capable of folding properly and exhibiting redox activity when expressed in the absence of the rest of the protein (9). In these studies, the N-terminal fragment included residues 1 through 202 based on the location of a tryptic-sensitive site between residues 202 and 203; this fragment was designated F[1202]. Reduction of F[1-202] by the separately expressed C-terminal fragment of AhpF, F[208-521], could be detected using DTNB as a reporter of electron transfer to F[1-202]. DTNB also served to reoxidize the $F[1-202]$ and sustain 
the electron-transfer reaction between fragments, mimicking its role in $\operatorname{TrR}$ assays with $\operatorname{Tr}(29)$.

When TrR was tested as a reductant of $F[1-202]$, the rate of electron transfer, at $2 \times 10^{4} \mathrm{M}^{-1} \mathrm{~s}^{-1}$, was about half that observed between $\mathrm{F}[208-521]$ and $\mathrm{F}$ [1-202] (9). By comparison, $k_{\text {cat }}$ and $k_{\text {cat }} / K_{\mathrm{m}}$ values for intact $\mathrm{AhpF}$ assayed with AhpC (at saturating levels of $\mathrm{NADH}$ and cumene hydroperoxide) are about $240 \mathrm{~s}^{-1}$ and $10^{7} \mathrm{M}^{-1} \mathrm{~s}^{-1}$ at $25^{\circ} \mathrm{C}$, respectively (30). Both flavoproteins ( $\operatorname{TrR}$ and $\mathrm{F}[208-521]$ ) show nonsaturable kinetics with $\mathrm{F}[1-202]$ in concentrations of the latter protein up to $100 \mu \mathrm{M}$. When TrR was tested with $F[1-202]$ at much lower concentrations of the flavoprotein (at 0.02 rather than $0.5 \mu \mathrm{M}$ TrR), the observed bimolecular rate constant for interaction between the two proteins was actually somewhat higher, at about $10^{5} \mathrm{M}^{-1}$ $\mathrm{s}^{-1}$. Thus, TrR is quite comparable to $\mathrm{F}[208-521]$ in its capacity to catalyze the reduction of the N-terminus of $\mathrm{AhpF}$ free in solution.

The midpoint potential for reduction of $\mathrm{F}$ [1-202] was also assessed and compared to that of $E$. coli $\operatorname{Tr}$ as a way of further defining the capacity of $F[1-202]$ to serve as a substrate for TrR. By evaluating the equilibrium mixture of reduced and oxidized proteins and pyridine nucleotides (NADPH and $\mathrm{NADP}^{+}$) in the presence of a small amount of $\mathrm{TrR}$, a value of $-0.264 \pm 0.008 \mathrm{~V}$ was obtained as the midpoint reduction potential of $\mathrm{F}[1-202]$. By comparison, the midpoint reduction potential for $\mathrm{Tr}$ as determined in these studies was very similar to that of $\mathrm{F}[1-202]$, at $-0.274 \pm$ $0.006 \mathrm{~V}$; this value compared favorably with that of -0.270 $\mathrm{V}$ determined previously using the same methods (24).

Interestingly, the $\mathrm{N}$-terminus of $\mathrm{AhpF}$ has recently been recognized to contain a tandem repeat of two Tr-like folds with the second of the two bearing the redox-active disulfide center $(9,18)$. Nonetheless, $F[1-202]$ is not recognized by TrR as a good substitute for $\mathrm{Tr}$; as described above, catalytic electron transfer between the two proteins is relatively slow and nonsaturable (the bimolecular rate constant for electron transfer between $\operatorname{TrR}$ and $\mathrm{F}[1-202]$, at $(2-10) \times 10^{4} \mathrm{M}^{-1}$ $\mathrm{s}^{-1}$, is several orders of magnitude lower than $k_{\text {cat }} / K_{\mathrm{m}}$ for electron transfer between $\operatorname{TrR}$ and $\mathrm{Tr}$, at about $4 \times 10^{7} \mathrm{M}^{-1}$ $\mathrm{s}^{-1}$ in our studies; see below). Furthermore, F[208-521] catalyzes electron transfer to $F[1-202]$ at a rate very comparable to that of $\mathrm{TrR}$, yet cannot reduce $\mathrm{Tr}$, even when the reaction is allowed to proceed for many hours (9). These studies have demonstrated a high degree of substrate specificity for F[208-521], the ability of TrR to substitute for $F[208-521]$ in assays for the reduction of free $F[1-$ 202], and a requirement for $\mathrm{F}[1-202]$ to be covalently attached to its physiological electron donor, F[208-521], for effective catalytic turnover.

Spectral Properties and Thiol Contents of Chimeric Flavoproteins Engineered with the N-Terminus of AhpF Linked to Full-Length TrR. The above results encouraged our pursuit of the construction of a chimeric protein, designated Nt-TrR, through linkage of the DNA encoding the N-terminal 207 amino acids of AhpF to that encoding TrR (Figure 2). This chimera was then evaluated for its ability to replace $\mathrm{AhpF}$ in $\mathrm{NAD}(\mathrm{P}) \mathrm{H}$-dependent peroxidase assays with $\mathrm{AhpC}$ and for its remaining TrR activity. As controls, we also constructed chimeric proteins with serine residues substituting for cysteine residues in each of the two potentially redox-active disulfide centers of the chimera (Nt-

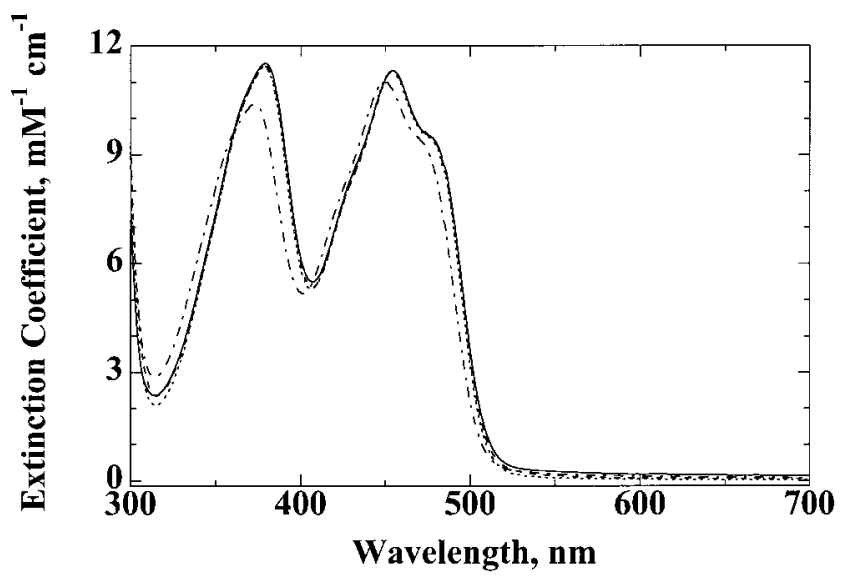

FIGURE 3: Visible absorbance spectra of TrR and Nt-TrR chimeric proteins. The proteins were in standard buffer at neutral $\mathrm{pH}$ and room temperature; spectra shown are for TrR (solid line), Nt-TrR (dotted line), Nt-TrR C129,132S (dashed line), and Nt-TrR C342,345S (dashed-dotted line).

\section{TrR C129,132S and Nt-TrR C342,345S).}

On expression and purification, it was clear that each of the three chimeric proteins, "wild type" Nt-TrR and the C129,132S or C342,345S mutants, could be obtained in good yield and possessed a tightly bound FAD cofactor with properties similar to those of the comparable TrR and AhpF proteins. Comparisons of the visible absorbance spectra of Nt-TrR and Nt-TrR C129,132S with that of their parent flavoprotein, TrR, indicated that all three proteins have virtually identical properties (Figure 3). Nt-TrR C342,345S, on the other hand, exhibits blue-shifted flavin peaks with maxima at 373 and $450 \mathrm{~nm}$ (compared with 379 and 454 $\mathrm{nm}$ for the other proteins) and a lowered extinction coefficient for the $373 \mathrm{~nm}$ absorbance band. The appearance of this spectrum is highly reminiscent of that previously published for the C138S mutant of TrR (29). This perturbation in the flavin spectrum of the C138S mutant of TrR was attributed to the presence of the serine hydroxyl group near the flavin rather than the disulfide bond normally present between Cys135 and Cys138 of TrR. Subsequent fluorescence (31) and reductive half-reaction (32) data have supported an altered equilibrium between two protein conformations in C138S with respect to wild-type TrR which is now believed to be partly responsible for the different spectral properties of the bound flavin in these proteins. Interestingly, although no spectra were shown for the mutant of TrR (C135,138S) most comparable to Nt-TrR C342,345S, that mutant was reported to possess properties more similar to the wild-type protein than to the C138S mutant of TrR (32). Attachment of the N-terminus from AhpF to that mutant of TrR may therefore have the effect of altering the equilibrium mixture of conformers and may provide an additional tool for characterizing the two putative conformations of $\operatorname{TrR}(16,17)$.

In addition to the appearance of the visible spectra, flavin contents and extinction coefficients of the bound FAD were essentially identical for Nt-TrR and Nt-TrR C129,132S. One tightly bound FAD per subunit in each protein (1.04 and 0.86 for Nt-TrR and Nt-TrR C129,132S, respectively) absorbs visible light with an extinction coefficient of 11300 $\mathrm{M}^{-1} \mathrm{~cm}^{-1}$ at $454 \mathrm{~nm}$ in both cases $\left(\epsilon_{454}=11300 \mathrm{M}^{-1} \mathrm{~cm}^{-1}\right.$ for $E$. coli TrR, as well). The single FAD bound to Nt-TrR 
C342,345S (0.86 per subunit) has an extinction coefficient of $11000 \mathrm{M}^{-1} \mathrm{~cm}^{-1}$ at $450 \mathrm{~nm}$ (Figure 3).

Given the different spectral properties observed for NtTrR C342,345S in comparison with Nt-TrR and Nt-TrR $\mathrm{C} 129,132 \mathrm{~S}$, and the possibility that that difference reflected altered conformational equilibria for that mutant, we also analyzed the flavin fluorescence of the three chimeric proteins and TrR. Previous studies by Mulrooney and Williams (31) had demonstrated a significantly quenched fluorescence spectrum for the C138S mutant when compared with wild-type $\operatorname{TrR}$ (7\% of the remaining fluorescence for the former compared with the latter protein). Indeed, NtTrR and Nt-TrR C129,132S exhibited identical fluorescence properties when compared with $\operatorname{TrR}\left(\lambda_{\max , \mathrm{ex}}=454 \mathrm{~nm}\right.$, $\left.\lambda_{\text {max,em }} \sim 516 \mathrm{~nm}\right)$, while the fluorescence of Nt-TrR C342,$345 \mathrm{~S}\left(\lambda_{\text {max,ex }}=450 \mathrm{~nm}, \lambda_{\text {max,em }} \sim 514 \mathrm{~nm}\right)$ was quenched to about $62 \%$ of the intensity compared with the other proteins. While the fluorescence quenching for Nt-TrR C342,345S is not as dramatic as that for the C138S mutant of TrR, this result is in qualitative agreement with the spectral perturbations observed for this mutant as described above.

Thiol contents of all chimeric proteins matched those expected based on previously reported values for wild-type TrR and AhpF proteins $(3,29)$. Oxidized Nt-TrR proteins all possessed approximately two free thiols per subunit (33); these correspond to the two noncatalytic cysteinyl residues present in TrR (29). Proteins incubated with excess NADPH, on the other hand, exhibited either six (5.89 \pm 0.15 per subunit for "wild type" Nt-TrR) or four ( $3.99 \pm 0.28$ or 3.77 \pm 0.21 per subunit, respectively, for Nt-TrR C129,132S and Nt-TrR C342,345S) thiols following reduction and denaturation. This result confirmed that the disulfide center in the appended N-terminus of Nt-TrR and Nt-TrR C342,345S is redox-active.

Reductive Titrations of Nt-TrR Proteins. Anaerobic titrations of Nt-TrR and the mutated control proteins with dithionite or NADPH were carried out to characterize spectral species and evaluate the presence and nature of redox-active disulfide centers in these proteins. Nt-TrR proteins exhibit spectral changes during reductive titrations that are similar to those observed during NADPH and dithionite titrations of TrR, as shown in Figures 4-6. Like TrR and AhpF, NtTrR proteins do not exhibit a detectable charge-transfer interaction between a nascent thiolate anion and oxidized FAD $(3,34)$. Addition of NADPH or dithionite leads to spectral changes indicative of the progressive reduction of FAD and the formation of the blue, neutral semiquinone form of the flavin with absorbance centered around $590 \mathrm{~nm}$ (Figures 4-6).

In the absence of light or EDTA, amounts of semiquinone stabilized during these titrations are low for Nt-TrR (Figures 4 and 5) and Nt-TrR C129,132S. The amount of blue, neutral semiquinone observed during reductive titrations varies with the mutant and with exposure to light and/or EDTA; this latter effect was previously reported for wild-type TrR (35). Exposure of the partially reduced samples to fluorescent room light does, however, lead to a reversible, light-induced increase in semiquinone stabilization for these two proteins. Interestingly, dithionite titration of Nt-TrR C342,345S leads to the stabilization of a very large amount of flavin semiquinone (around 90\%) even in the absence of light and EDTA (Figure 6). The extinction coefficient of this species
A

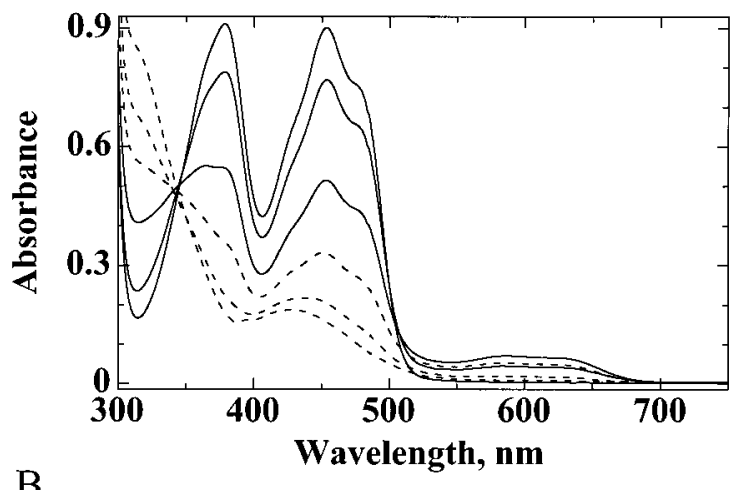

B

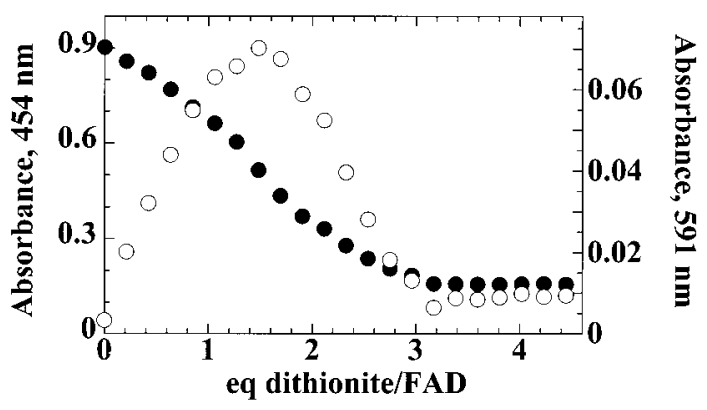

FIGURE 4: Anaerobic dithionite titration of Nt-TrR. The titration was carried out in $25 \mathrm{mM}$ potassium phosphate buffer (lacking EDTA) at pH 7.0 in a total volume of $600 \mu \mathrm{L}$ at $25^{\circ} \mathrm{C}$ in the dark. $\mathrm{Nt}-\operatorname{TrR}(48.5 \mathrm{nmol})$ was titrated in the presence of $0.5 \mathrm{nmol}$ of methyl viologen with a $7.9 \mathrm{mM}$ solution of dithionite. Spectra were recorded after each addition when no further absorbance changes occurred. Solid lines in panel A represent spectra obtained after the addition of $0,0.64$, and 1.48 equiv of dithionite/FAD in order of decreasing $A_{454}$ and increasing $A_{591}$. Dashed lines in panel A indicate spectra obtained after the addition of 2.12, 2.75, and 3.39 equiv of dithionite/FAD in order of decreasing $A_{454}$ and $A_{591}$. Panel $\mathrm{B}$ shows the absorbance changes at 454 (closed circles) and 591 $\mathrm{nm}$ (open circles) versus equiv of dithionite/FAD added.

( $4500 \mathrm{M}^{-1} \mathrm{~cm}^{-1}$ ), obtained by the extrapolation of $A_{450}$ and $A_{590}$ data, is very similar to those values reported for wildtype TrR (using photoreduction) and $\operatorname{AhpF}(3,35)$. By comparison, dithionite titrations of Nt-TrR and Nt-TrR C129,132S in the absence of light and EDTA lead to the stabilization of, at most, $20 \%$ semiquinone (based on the above extinction coefficient). This characteristic of stabilizing large amounts of flavin semiquinone therefore distinguishes Nt-TrR C342,345S from the other two chimeric proteins, as do the absorbance and fluorescence properties of the bound flavin described above (Figure 3), further supporting the interpretation that this mutant chimeric protein favors a different equilibrium mixture of conformers than do the other two chimeras and wild-type TrR (see above).

Continued additions of dithionite during these reductive titrations (Figures 4 and 6) lead to full reduction of the flavin in all three Nt-TrR constructs. NADPH, on the other hand, is not as effective a reductant of the flavin semiquinone for these chimeric proteins or TrR (Figure 5) (34). NADPH titrations, but not dithionite titrations, result in the development of a long-wavelength charge-transfer band in Nt-TrR. This absorbance is the hallmark of electronic interaction between $\mathrm{FADH}_{2}$ and $\mathrm{NADP}^{+}(36)$ and may be characteristic of a redox species important in catalysis by $\operatorname{TrR}(37)$ as well as Nt-TrR. The spectrum of this species for Nt-TrR (Figure 5) suggests a much shorter $\lambda_{\max }$ (below $700 \mathrm{~nm}$ ) than is 
A

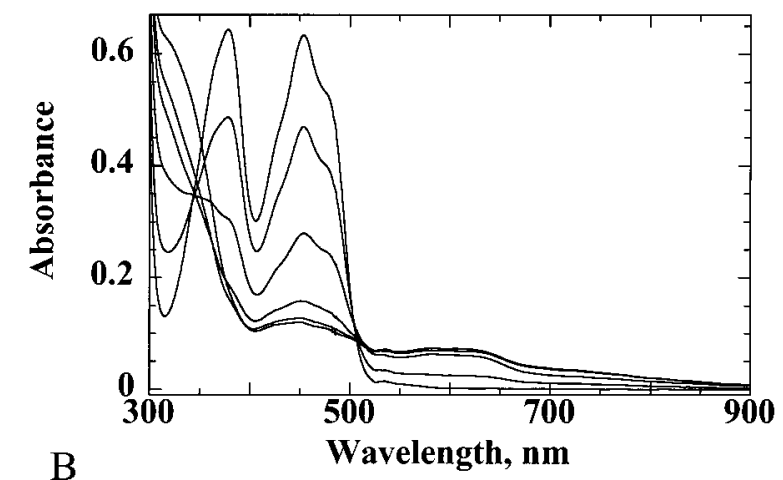

$\mathrm{B}$

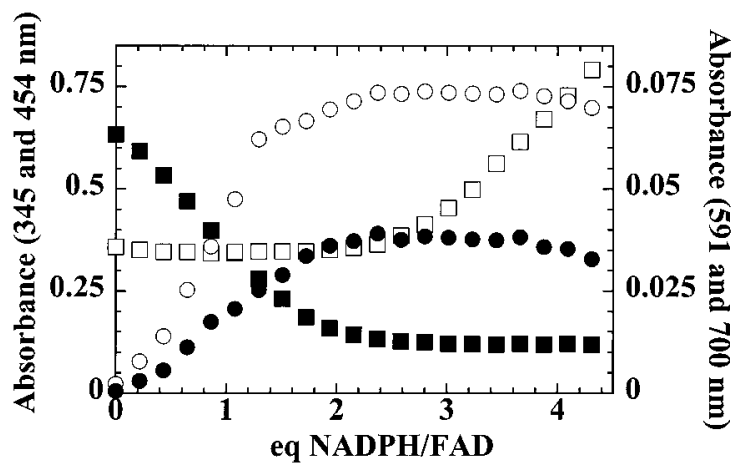

FIGURE 5: Anaerobic NADPH titration of Nt-TrR. The titration was carried out using the same conditions as in Figure 4, but with a total volume of $500 \mu \mathrm{L}$. Nt-TrR $(28.3 \mathrm{nmol})$ was titrated with a $4.7 \mathrm{mM}$ anaerobic solution of NADPH. Spectra were recorded after each addition when no further absorbance changes occurred. Solid lines in panel A indicate spectra obtained after the addition of 0 , $0.65,1.29,1.94,2.58$, and 3.23 equiv of NADPH/FAD in order of decreasing $A_{454}$. Panel B depicts the absorbance changes at 345 (open squares), 454 (closed squares), 591 (open circles), and 700 $\mathrm{nm}$ (closed circles) versus equiv of NADPH/FAD. Intersection of the linear portions of the $345 \mathrm{~nm}$ absorbance changes (near the isosbestic points between $\mathrm{FAD}$ and $\mathrm{FADH}^{\bullet}$ or $\mathrm{FADH}_{2}$ ) indicates oxidation of 2.7 equiv of NADPH/FAD.

generally observed for the $\mathrm{FADH}_{2} \rightarrow \mathrm{NAD}^{+}$charge-transfer absorbance band of AhpF and its relatives $\left(\lambda_{\max } \sim 730-\right.$ $760 \mathrm{~nm})(3,30)$, indicating a higher energy for interaction between the two cofactors in this case.

The evaluation of the stoichiometry of reductant addition which leads to the observed spectral changes as described above for the chimeric proteins is also very informative with regard to the number of redox centers undergoing reduction. In both dithionite and NADPH titrations of Nt-TrR, 1 extra equiv per subunit of each reductant is required when compared with similar titrations of TrR, Nt-TrR C129,132S, or Nt-TrR C342,345S. As shown in panel B of Figure 4, dithionite titrations require about 3 equiv/FAD of the reductant to achieve complete reduction of Nt-TrR relative to about 2 equiv/FAD for TrR or Nt-TrR C129,132S (33, $34)$. The requirement of 3 equiv/FAD for full reduction of $\mathrm{Nt}-\mathrm{TrR}$ by dithionite, as was observed previously for $\mathrm{AhpF}$ (3), along with the thiol content of about 6 per subunit for the reduced protein (see above), confirms the presence of two disulfide redox centers in addition to the flavin.

The attachment of the AhpF N-terminus to TrR also changes the stoichiometry of reductant which leads to maximal flavin semiquinone formation as compared with TrR. While maximal semiquinone formation occurs at $\sim 0.7$
A

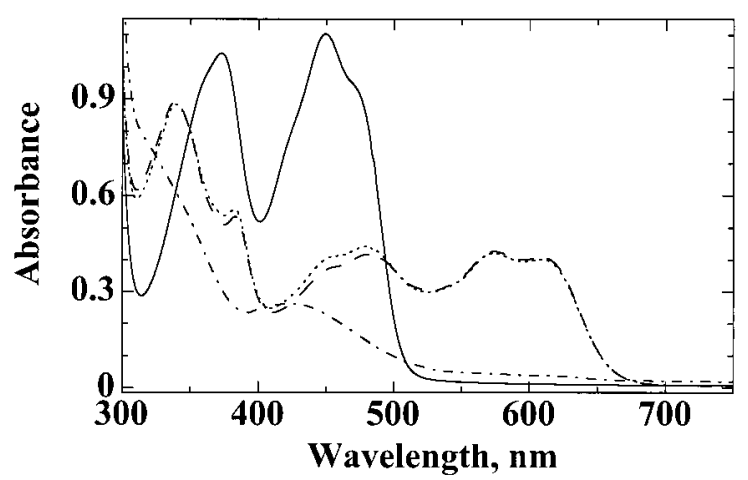

$\mathrm{B}$

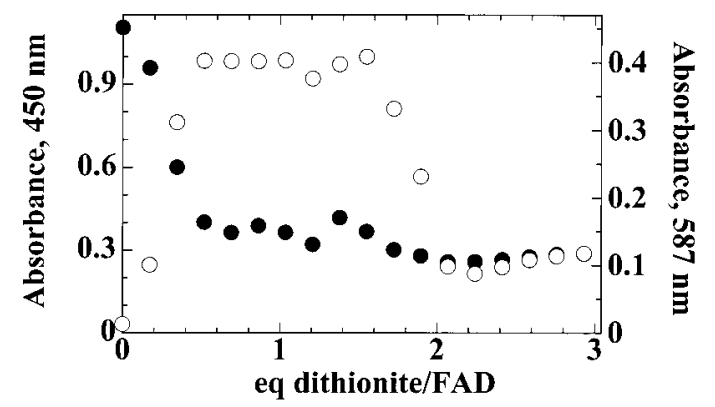

FIGURE 6: Anaerobic dithionite titration of Nt-TrR C342,345S. The titration was carried out as in Figure 4, except that Nt-TrR C342,345S $(61.1 \mathrm{nmol})$ was titrated in the presence of $6 \mathrm{nmol}$ of methyl viologen with a $8.13 \mathrm{mM}$ dithionite solution. Spectra in panel A were obtained after the addition of 0 (solid line), 0.52 (dotted line), 1.55 (dashed line), and 2.07 (dashed-dotted line) equiv of dithionite/FAD. The final spectrum (dashed-dotted line) was corrected for the spectral contribution of the relatively high level of reduced methyl viologen present. Panel B shows the absorbance changes at 454 (closed circles) and $591 \mathrm{~nm}$ (open circles) versus equiv of dithionite/FAD added.

equiv/FAD for TrR or Nt-TrR C129,132S (33, 34), Nt-TrR exhibits maximal semiquinone formation at $\sim 1.5$ equiv/FAD (Figure 4). Interestingly, dithionite titrations of Nt-TrR C342,345S also require about 2 equiv/FAD to complete the titration (Figure 6), and maximal semiquinone is maintained from about $0.5-1.6$ equiv of dithionite/FAD. This result, along with the thiol content of reduced Nt-TrR C342,345S of about 4 per subunit, indicates that electron transfer from the reduced flavin to the N-terminal disulfide center can occur in the absence of the Cys342-Cys345 center. This observation is in accordance with the results of similar titrations with the corresponding mutant of $\mathrm{AhpF}, \mathrm{AhpF}$ C345,348S (8). Titrations of both of these mutants require relatively long equilibration times after each addition, indicating that the normal pathway of electron transfer has been disrupted.

NADPH titrations of Nt-TrR, as shown in Figure 5, are reminiscent of NADH titrations of $\mathrm{AhpF}$ in requiring $>2.5$ equiv/FAD of the pyridine nucleotide (3). Oxidation of NADPH monitored at $345 \mathrm{~nm}$, where the conversion of oxidized flavin to $\mathrm{FADH}_{2}$ and semiquinone is approximately isosbestic and NADPH absorbs strongly, accounts for 2.7 equiv/FAD (Figure 5B), rather than about 1.6 equiv/FAD as measured for TrR or Nt-TrR C129,132S (data not shown), again consistent with the presence of the additional $\mathrm{N}$ terminal redox center in Nt-TrR.

Analytical Ultracentrifugation Studies of Nt-TrR. Given that both $\mathrm{TrR}$ and $\mathrm{AhpF}$ have been characterized as dimeric 
Table 1: DTNB Reductase and AhpC-Linked Peroxidase Activities of AhpF, TrR, and Chimeric Proteins ${ }^{a}$

\begin{tabular}{lccccc}
\hline & \multicolumn{4}{c}{ protein analyzed } \\
\cline { 2 - 5 } activity catalyzed & AhpF & TrR & Nt-TrR & Nt-TrR C129,132S & Nt-TrR C342,345S \\
\hline $\begin{array}{l}\text { DTNB reductase activity } \\
\text { peroxidase activity }\end{array}$ & $966 \pm 96$ & $4.8 \pm 0.4$ & $655 \pm 60$ & $4.3 \pm 0.6$ & NA $^{c}$ \\
$\quad k_{\text {cat }}{ }^{e}$ & $204 \pm 16$ & NA & $50.3 \pm 1.5$ & NA & NA \\
$K_{\mathrm{m}}{ }^{\text {AhpC } f}$ & $15.2 \pm 3.1$ & & $4.8 \pm 0.5$ & & \\
$k_{\text {cat }} / K_{\mathrm{m}}{ }^{g}$ & $1.3 \times 10^{7}$ & & $1.0 \times 10^{7}$ & & \\
\hline
\end{tabular}

${ }^{a}$ Values reported as mean \pm standard error. ${ }^{b}$ DTNB reductase activity was measured aerobically in $50 \mathrm{mM}$ Tris-HCl, pH 8.0, $0.5 \mathrm{mM}$ EDTA, $50 \mu \mathrm{M}$ NAD $(\mathrm{P}) \mathrm{H}$, and $500 \mu \mathrm{M}$ DTNB, in a total volume of $1.0 \mathrm{~mL}$ at $25^{\circ} \mathrm{C}$; values expressed as $\mu$ mol of DTNB consumed min ${ }^{-1}(\mu \mathrm{mol}$ bound FAD $)^{-1} \cdot{ }^{c} \mathrm{NA}=$ no activity. ${ }^{d}$ Peroxidase activity was measured aerobically in $50 \mathrm{mM}$ potassium phosphate, $\mathrm{pH} 7.0,0.5 \mathrm{mM}$ EDTA, $100 \mathrm{mM}$ ammonium sulfate, $1 \mathrm{mM}$ cumene hydroperoxide, and $150 \mu \mathrm{M} \mathrm{NAD}(\mathrm{P}) \mathrm{H}$ at $25^{\circ} \mathrm{C}$; these experiments employed a stopped-flow spectrophotometer for data collection. Rates were adjusted by subtracting the very low rate of $\mathrm{NAD}(\mathrm{P}) \mathrm{H}$ consumption by the flavoproteins in the absence of AhpC. Kinetic data were fit to the Michaelis-Menten equation using the ENZFITTER program. ${ }^{e}$ Values expressed as $\mu$ mol of NAD(P)H consumed s ${ }^{-1}$ ( $\mu \mathrm{mol}$ of bound FAD) $)^{-1} \cdot{ }^{f} K_{\mathrm{m}}{ }^{\text {AhpC }}$ expressed as $\mu \mathrm{M} .{ }^{g}$ Values expressed as $\mathrm{M}^{-1} \mathrm{~s}^{-1}$.

proteins $(9,17)$, we used sedimentation equilibrium analyses to characterize the oligomerization state of Nt-TrR. Analyses of individual data sets at a variety of concentrations and speeds yielded molecular weight values that exhibited no significant dependence on either protein concentration or rotor speed, consistent with the single ideal species model. Global fits of the data to this model resulted in a weightaverage $M_{\mathrm{w}}$ of $111300 \pm 1900$ (see Supporting Information for a plot of representative data). The theoretical $M_{\mathrm{w}}$ of an Nt-TrR monomer is 57225 . Thus, a dimer would have a calculated $M_{\mathrm{w}}$ of 114450 . These analytical ultracentrifugation studies show that Nt-TrR is a dimer under the conditions of this experiment. Attachment of the N-terminal portion of AhpF to TrR does not, therefore, alter the ability of the TrRderived part of the protein to dimerize. In previous studies, both intact AhpF and the F[208-521] fragment were shown to be dimers, while $\mathrm{F}[1-202]$ was itself monomeric, even at high concentration (9). Dimerization of intact AhpF and $\mathrm{Nt}-\mathrm{TrR}$ may be a requirement for catalysis of $\mathrm{AhpC}$ reduction if electron transfer between the $\mathrm{C}$-terminal and $\mathrm{N}$-terminal dithiol/disulfide centers is an intersubunit process, as has been hypothesized previously (9).

$N A D(P) H$-Dependent DTNB and AhpC-Linked Peroxide Reductase Activities of AhpF, TrR, and Chimeric Proteins. The efficiency of AhpF in catalyzing the reduction of the disulfide bond of DTNB has been demonstrated previously (3). TrR, on the other hand, cannot efficiently reduce DTNB in the absence of $\operatorname{Tr}(29)$. Nt-TrR C129,132S, like TrR, is unable to transfer electrons efficiently to DTNB in the absence of Tr; both Nt-TrR C129,132S and the parent protein, TrR, exhibit activities $<0.5 \%$ that of $\mathrm{AhpF}$ (Table 1). The other mutant chimera, Nt-TrR C342,345S, has no detectable DTNB reductase activity. Under these conditions, however, Nt-TrR exhibits about $70 \%$ of the activity of wildtype AhpF. Attachment of the N-terminal portion of AhpF to TrR clearly imparts high NADPH-dependent DTNB reductase activity to this protein as long as the redox-active disulfide center remains intact, indicating that the dithiol form of the N-terminal redox center is the main site of DTNB reactivity in Nt-TrR.

Flavoprotein-dependent peroxidase assays carried out aerobically in the presence of saturating substrates $[150 \mu \mathrm{M}$ $\mathrm{NAD}(\mathrm{P}) \mathrm{H}$ and $1 \mathrm{mM}$ cumene hydroperoxide] also indicated that Nt-TrR is highly efficient at reducing AhpC. Unlike TrR, Nt-TrR has significant AhpC-linked peroxidase activity as long as the N-terminal disulfide center is intact; the value of $k_{\text {cat }}$ for Nt-TrR is about $25 \%$ that of AhpF (Table 1). NtTrR also has a considerably lower $K_{\mathrm{m}}$ for AhpC than does AhpF; in part because of this lower $K_{\mathrm{m}}$, the catalytic efficiencies $\left(k_{\text {cat }} / K_{\mathrm{m}}\right)$ for Nt-TrR and AhpF are essentially equal, at around $10^{7} \mathrm{M}^{-1} \mathrm{~s}^{-1}$. Thus, as with DTNB reductase activity assays, catalytic reduction of AhpC is not catalyzed by $\operatorname{TrR}$ and is only catalyzed by chimeric Nt-TrR proteins if both redox-active disulfide centers are intact.

To examine the possibility that the considerable peroxidase activity of Nt-TrR is a result of intermolecular electron transfer between Nt-TrR dimers, equimolar amounts of the two different disulfide-center mutants of Nt-TrR, Nt-TrR C129,132S and Nt-TrR C342,345S, were mixed in the presence of AhpC and assayed for peroxidase activity. If the peroxidase activity of Nt-TrR is a result of intermolecular electron transfer, we would expect a mixture of these mutants to exhibit $50 \%$ of the $k_{\text {cat }}$ and the same $K_{\mathrm{m}}$ when compared with Nt-TrR due to the presence of half the total number of normal reactive disulfide centers. On mixing these mutants at high concentrations (2.5 or $5 \mu \mathrm{M}$ of each protein), however, we observed no detectable peroxidase activity, indicating that intermolecular electron transfer between dimers is not involved in the catalysis of AhpC reduction.

Insulin-Linked TrR Activities of AhpF, TrR, and Chimeric Proteins. Although AhpF is clearly a homologue of $\operatorname{TrR}$, neither AhpF nor F[208-521] is efficient at reducing $\operatorname{Tr}(9)$. In the context of the present studies, however, it was of considerable interest to assess the ability of $\mathrm{Nt}-\mathrm{TrR}$ to reduce $\mathrm{Tr}$ given the additional large domain attached to the parent protein in this case. We chose to use insulin-linked assays to examine the reduction of Tr by Nt-TrR, instead of DTNBlinked assays, due to the inherent DTNB reductase activity of Nt-TrR.

As shown in Table 2, Nt-TrR is quite efficient at reducing $\operatorname{Tr}$ [the values of $k_{\text {cat }}$ and $K_{\mathrm{m}}$ determined for wild-type $\operatorname{TrR}$ in this study were slightly lower than the previously reported values of $33 \mathrm{~s}^{-1}$ and $2.8 \mu \mathrm{M}$, respectively; (38)]. The turnover number of Nt-TrR is $86 \%$ that of the TrR protein. Also, the $K_{\mathrm{m} \text {,app }}$ for $\operatorname{Tr}$ is quite similar for both proteins. Nt-TrR $\mathrm{C} 129,132 \mathrm{~S}$ is also quite efficient at reducing $\operatorname{Tr}\left(k_{\mathrm{cat}}\right.$ is $71 \%$ that of $\operatorname{TrR}$, and $K_{\mathrm{m} \text {,app }}$ values for $\operatorname{Tr}$ are comparable), indicating that mutagenesis of the Cys129-Cys132 center has essentially no effect on the protein's ability to reduce Tr. Disruption of the other catalytic disulfide center in the C342,345S mutant of Nt-TrR, in contrast, abolishes all TrR 
Table 2: Comparison of Insulin-Linked Tr Reduction Activities of AhpF, TrR, and Chimeric Proteins ${ }^{a}$

\begin{tabular}{lccccc}
\hline & \multicolumn{5}{c}{ protein analyzed } \\
\cline { 2 - 6 } steady-state \\
\cline { 2 - 6 } parameter & AhpF & TrR & Nt-TrR & C129,132S & C342,345S \\
\hline$k_{\text {cat }}^{b}$ & NA & $27.6 \pm 1.3$ & $23.6 \pm 1.0$ & $19.6 \pm 0.8$ & NA $^{c}$ \\
$K_{\mathrm{m}}{ }^{T r} d$ & & $0.7 \pm 0.1$ & $1.0 \pm 0.2$ & $1.0 \pm 0.2$ & \\
$k_{\text {cat }} / K_{\mathrm{m}}{ }^{e}$ & & $4.2 \times 10^{7}$ & $2.3 \times 10^{7}$ & $2.0 \times 10^{7}$ & \\
\hline
\end{tabular}

${ }^{a}$ Insulin-linked Tr reduction assays were carried out aerobically in $50 \mathrm{mM}$ potassium phosphate, $\mathrm{pH}$ 7.0, $1 \mathrm{mM}$ EDTA, $150 \mu \mathrm{M}$ NADPH and $100 \mu \mathrm{M}$ bovine pancreas insulin, in a total volume of $0.5 \mathrm{~mL}$ at $25^{\circ} \mathrm{C}$. Values given as mean \pm standard error. ${ }^{b}$ Values expressed as $\mu$ moles $\mathrm{NAD}(\mathrm{P}) \mathrm{H}$ consumed $\mathrm{s}^{-1} \mu$ mole $^{-1}$ bound FAD. ${ }^{c} \mathrm{NA}=\mathrm{No}$ activity. ${ }^{d}$ Values expressed in $\mu \mathrm{M} .{ }^{e}$ Values expressed in $\mathrm{M}^{-1} \mathrm{~s}^{-1}$.

activity as would be expected if this center is the site of $\mathrm{Tr}$ reduction.

Other Studies of Chimeric or Fusion Proteins. Efforts to link pyridine nucleotide-dependent flavoprotein reductases with their small redox protein substrates have been successful in other cases, as well. Several classic cases of such fusion proteins are cytochrome $\mathrm{P} 450 / \mathrm{NADPH}-$ cytochrome $\mathrm{P} 450$ reductase fusion proteins $(39-41)$ and the ferredoxin/ ferredoxin $-\mathrm{NADP}^{+}$reductase $(\mathrm{Fd} / \mathrm{FNR})$ fusion protein (42). In each of these cases, design of the fusion proteins was guided by naturally occurring fusions of related proteins. For example, a single polypeptide possessing both the cytochrome P450 and its reductase is expressed in bacterial species (43). In the case of ferredoxin $(\mathrm{Fd})$, precedent for attachment of this $[2 \mathrm{Fe}-2 \mathrm{~S}]$ cluster-containing protein at either the N-terminal or the C-terminal end of the FNR family member has been observed $[\mathrm{Fd}$, then reductase in phthalate oxygenase reductase (44); and reductase, then $\mathrm{Fd}$ in xylene monooxygenase reductase (45)]. The construction of the $\mathrm{Fd} /$ FNR fusion was especially guided by the knowledge of the structure of spinach FNR $(46,47)$, where 18 residues at the N-terminus are not visible in the X-ray structure and Tyr314 at the C-terminus is in the active site and likely to play a role in catalysis. Interestingly, the $\mathrm{Fd} / \mathrm{FNR}$ fusion protein clearly mimicked the dissociable, but stable complex normally observed between the two proteins from spinach, and electron transfer between the two segments of Fd/FNR was a highly efficient, intramolecular process (42). Our results with Nt-TrR have also demonstrated intramolecular electron transfers between the two segments which are much more efficient in the chimera than in the separate proteins/ fragments.

A recently identified fusion protein between $\operatorname{TrR}$ and $\operatorname{Tr}$ expressed by Mycobacterium leprae represents a system potentially much more closely related to Nt-TrR described herein $(48,49)$. This protein, with $\operatorname{Tr}$ attached at the C-terminal rather than the N-terminal end of TrR, exhibits both activities. It was not clear, however, whether electron transfer between $\operatorname{TrR}$ and $\operatorname{Tr}$ in this fusion protein is an intraor intermolecular process.

Our studies of Nt-TrR are a little different from the previous studies described above in that the N-terminal domain of AhpF does not function as a highly active redox carrier when expressed independently (9). This protein "module" has also not been identified in any organism as a separately expressed protein. It is clear, however, that attachment of this module imparts high $\mathrm{AhpC}$ reductase activity to TrR which does occur as an intramolecular process. As the $\mathrm{N}$ - and $\mathrm{C}$-termini of $\mathrm{TrR}$ are quite close to one another (17), the architecture of Nt-TrR may not be all that different from the $\mathrm{TrR}-\mathrm{Tr}$ fusion protein expressed in M. leprae. Crystallographic studies of both AhpF and NtTrR are currently underway to improve our understanding of how the N-terminal domain of AhpF interacts with the flavoprotein reductase part of these proteins to effect catalysis of AhpC reduction (Z. Wood and P. A. Karplus, unpublished).

Implications for the Multiple Activities of Nt-TrR. In terms of catalytic efficiency, Nt-TrR is as good a catalyst of AhpC reduction as is $\mathrm{AhpF}$. The modest decrease in the turnover number for $\mathrm{AhpC}$ reduction by $\mathrm{Nt}-\mathrm{TrR}$ is compensated for by the accompanying decrease in the $K_{\mathrm{m}}$ for AhpC. Thus, although Nt-TrR is an "unnatural" enzyme created by synthetic means, it is a highly effective catalyst with some alterations in kinetic parameters that will require further investigation. An advantage to using Nt-TrR in place of $\mathrm{AhpF}$ in some experiments is its essentially complete lack of oxidase activity, unlike AhpF (2). For example, assays of crude bacterial extracts for AhpC activity using excess flavoprotein are much more straightforward and sensitive using Nt-TrR than using AhpF or its counterpart from Streptococcus mutans, Nox-1, which has an even higher oxidase activity $(30,50)$. It is notable that the flavoproteinassociated properties of Nt-TrR (e.g., specificity for NADPH rather than $\mathrm{NADH}$ as reductant, low oxidase and transhydrogenase activities, and high flavin fluorescence) closely follow those characteristics of the parental TrR protein, while the new activities observed for Nt-TrR (AhpC-dependent peroxidase and DTNB reductase activities) are characteristic of intact $\mathrm{AhpF}$ with a functional N-terminal disulfide center $(2,3,8)$. In all AhpF mutants characterized to date, DTNB reductase activities have paralleled AhpC-linked peroxidase activities, indicating a direct role for the $\mathrm{N}$-terminal domain in both activities.

While our demonstration that Nt-TrR is a highly active AhpC reductase was quite a gratifying result, the retention of essentially full TrR activity by this chimera was a more surprising result. We reasoned that, if the N-terminus of AhpF attached to TrR was capable of interacting with the Cys342-Cys345 active site (originally Cys135 and Cys138, respectively, of $\mathrm{TrR}$ ) for electron transfer, then this large domain of about 200 amino acids would be likely to block access for $\operatorname{Tr}$ to approach this same active site. The high TrR activity observed for Nt-TrR argues against such a blockage by the $\mathrm{N}$-terminal domain, however. An alternative interpretation, that the $\mathrm{N}$-terminal domain is itself the donor of electrons to $\mathrm{Tr}$ in Nt-TrR, is not likely for several reasons: (i) both $\mathrm{Nt}-\mathrm{TrR}$ proteins with intact Cys342Cys 345 centers have essentially the same TrR activity (nearly identical $k_{\text {cat }}$ and $K_{\mathrm{m}}$ values) as wild-type TrR whether the N-terminal redox center is intact, and (ii) the midpoint reduction potentials for free $\mathrm{F}$ [1-202] (the separately expressed $\mathrm{N}$-terminal domain of $\mathrm{AhpF}$ ) and $\mathrm{Tr}$ are about the same. One implication of this high TrR activity for Nt-TrR, then, is that the N-terminal domain must be attached to the rest of the protein via a very long and mobile linker which is capable of moving this domain into proximity of Cys342Cys345 for electron transfer and also to a distal position such that $\mathrm{Tr}$ can gain access to this same redox center. Presumably 
the latter position of the N-terminal domain would also be one in which the Cys129-Cys132 active site is most accessible for direct electron transfer to the large substrate protein, AhpC (18).

Another implication of the high TrR activity of Nt-TrR has to do with the multiple types of conformational changes that are expected to occur in order to bring the different pairs of redox centers together in AhpF. Previous studies of TrR suggest that that protein alternates between two conformations during catalysis. One conformation, initially observed by X-ray crystallography (17), has the active site disulfide center in the pyridine nucleotide binding domain located close to the isoalloxazine ring of the flavin bound to the other domain (the FO conformation). The other, recently observed by crystallographic studies of a combined $\mathrm{TrR}-\mathrm{Tr}$ construct (16), has the pyridine nucleotide binding domain swung around about $66^{\circ}$ with respect to the flavin domain, to bring the nicotinamide ring of $\mathrm{NADP}^{+}$and the isoalloxazine ring of the flavin into a stacked arrangement and the Cys135Cys 138 center out to a more exposed position for interaction with $\operatorname{Tr}$ (the FR conformation). Our hypothesis has been that the N-terminal domain of AhpF, similar to $\mathrm{Tr}$, moves into position for electron transfer when the rest of $\mathrm{AhpF}$ is in the FR conformation and moves away from this part of $\mathrm{AhpF}$ for interaction with AhpC when the rest of the protein is in the FO conformation, like TrR with a tethered substrate (the $\mathrm{N}$-terminal domain). This proposal suggests a coordination between the two parts of AhpF that, if it exists, is apparently not obligatory. Thus, the TrR part of Nt-TrR is still fully capable of the FR $\leftrightarrow$ FO conformational changes required for $\mathrm{Tr}$ reduction, even though the $\mathrm{N}$-terminus must be staying out of the way during turnover with Tr. This discovery has been possible due to the additional, $\mathrm{N}$-terminus-independent, C-terminal disulfide-dependent activity exhibited by $\operatorname{TrR}$ in the chimeric construct (the comparable C-terminal fragment of AhpF has no such TrR activity).

Finally, an overview of all the results from these and AhpF fragment studies supports a view of $\mathrm{AhpF}$ as a highly efficient and specific reactivation system for $\mathrm{AhpC}$ that has evolved in a subset of the various species of eubacteria (30). In cases where AhpF is not expressed but the alternative reactivation system for the $\mathrm{AhpC}$ (peroxiredoxin) homologue has been identified, at least two or more redox components are involved in the pyridine nucleotide-dependent reactivation process [e.g., TrR and $\operatorname{Tr}$ in yeast (50), and trypanothione reductase, trypanothione, and tryparedoxin in Crithidia fasciculata (51)]. Unpublished work by D. Ritz, F. Aslund, and J. Beckwith has demonstrated that $\mathrm{AhpF}$ is not functional as a general reductant of protein disulfide bonds. Overexpression of $\mathrm{AhpF}$ and $\mathrm{AhpC}$ was not capable of restoring the appropriate redox balance to $E$. coli mutants lacking $\operatorname{tr} x B$ and gor (the structural gene for glutathione reductase). AhpF is therefore an efficient reactivator of AhpC (with $k_{\text {cat }} / K_{\mathrm{m}}$ for AhpC at about $10^{7} \mathrm{M}^{-1} \mathrm{~s}^{-1}$ ) with little or no capacity to reduce other proteins.

Considering the specificity of AhpF for AhpC but not $\mathrm{Tr}$, it is interesting to note that, were the C-terminal part of $\mathrm{AhpF}$ capable of Tr reduction, the pool of reduced Tr in cells would presumably be maintained at the expense of both NADH and NADPH. As it is, however, NADPH is the source of electrons for the redox homeostasis-maintaining flavoproteins glutathione reductase and $\mathrm{TrR}$, while the oxidative stress responsive alkyl hydroperoxide reductase system predominantly utilizes electrons from $\mathrm{NADH}$ to eliminate hydroperoxide-containing species. The $\mathrm{H}_{2} \mathrm{O}_{2}$-activated OxyR transcriptional regulator provides "cross-talk" between the redox state of cells [through glutaredoxin-1(red)/glutaredoxin-1(ox) and GSH/GSSG ratios] and their need for an oxidative stress response (through direct sensing of $\mathrm{H}_{2} \mathrm{O}_{2}$ levels); the OxyR regulon includes such proteins as glutathione reductase, glutaredoxin-1, thioredoxin-2, AhpF, and AhpC. Shortcircuiting this delicately balanced system through overlapping enzyme activities (high AhpC-reducing activity for TrR and $\mathrm{Tr}$, or high TrR activity for AhpF) could thus be quite disruptive to the normal survival mechanisms for these bacteria.

\section{ACKNOWLEDGMENT}

We thank Cindy Delong for initial optimization of the megaprimer PCR conditions used to generate $n t-\operatorname{tr} x B$.

\section{SUPPORTING INFORMATION AVAILABLE}

A figure depicting the sedimentation equilibrium data for Nt-TrR (Figure S1) is included as Supporting Information (1 page). This material is available free of charge via the Internet at http://pubs.acs.org.

\section{REFERENCES}

1. Jacobson, F. S., Morgan, R. W., Christman, M. F., and Ames, B. N. (1989) J. Biol. Chem. 264, 1488-1496.

2. Poole, L. B., and Ellis, H. R. (1996) Biochemistry 35, 5664.

3. Poole, L. B. (1996) Biochemistry 35, 65-75.

4. Ellis, H. R., and Poole, L. B. (1997) Biochemistry 36, 1334913356.

5. Storz, G., Tartaglia, L. A., Farr, S. B., and Ames, B. N. (1990) Trends Genet. 6, 363-368.

6. Tartaglia, L. A., Storz, G., Brodsky, M. H., Lai, A., and Ames, B. N. (1990) J. Biol. Chem. 265, 10535-10540.

7. Chae, H. Z., Robison, K., Poole, L. B., Church, G., Storz, G., and Rhee, S. G. (1994) Proc. Natl. Acad. Sci. U.S.A. 91, 70177021.

8. Li Calzi, M., and Poole, L. B. (1997) Biochemistry 36, 1335713364.

9. Poole, L. B., Godzik, A., Nayeem, A., and Schmitt, J. D. (2000) Biochemistry 39, 6602-6615.

10. Poole, L. B. (1999) in Flavins and Flavoproteins 1999 (Ghisla, S., Kroneck, P., Macheroux, P., and Sund, H., Eds.) pp 691694, Agency for Scientific Publications, Berlin.

11. Williams, C. H., Jr. (1995) FASEB J. 9, 1267-1276.

12. Holmgren, A. (1989) J. Biol. Chem. 264, 13963-13966.

13. Prinz, W. A., Aslund, F., Holmgren, A., and Beckwith, J. (1997) J. Biol. Chem. 272, 15661-15667.

14. Gladyshev, V. N., Jeang, K. T., and Stadtman, T. C. (1996) Proc. Natl. Acad. Sci. U.S.A. 93, 6146-6151.

15. Arscott, L. D., Gromer, S., Schirmer, R. H., Becker, K., and Williams, C. H., Jr. (1997) Proc. Natl. Acad. Sci. U.S.A. 94, $3621-3626$.

16. Williams, C. H., Jr., Arscott, L. D., Muller, S., Lennon, B. W., Ludwig, M. L., Wang, P.-F., Becker, K., and Schirmer, R. H. (2000) Eur. J. Biochem. (in press).

17. Waksman, G., Krishna, T. S. R., Williams, J., C. H., and Kuriyan, J. (1994) J. Mol. Biol. 236, 800-816.

18. Poole, L. B., Reynolds, C. M., Wood, Z., Karplus, P. A., Ellis, H. R., and Li Calzi, M. (2000) Eur. J. Biochem. (in press).

19. Higuchi, R. (1990) PCR protocol: a guide to methods and applications, Academic Press, San Diego.

20. Gally, D. L., Rucker, T. J., and Blomfield, I. C. (1994) J. Bacteriol. 176, 5665-5672. 
21. Parsonage, D., Miller, H., Ross, R. P., and Claiborne, A. (1993) J. Biol. Chem. 268, 3161-3167.

22. Ausubel, F. M., Brent, R., Kingston, R. E., Moore, D. D., Seidman, J. G., Smith, J. A., and Struhl, K. (1992) Short Protocols in Molecular Biology, 2nd ed., John Wiley and Sons, New York.

23. Lennon, B. W., and Williams, C. H., Jr. (1995) Biochemistry 34, 3670-3677.

24. Krause, G., Lundstrom, J., Barea, J. L., Pueyo de la Cuesta, C., and Holmgren, A. (1991) J. Biol. Chem. 266, 9494-9500.

25. Clark, W. M. (1960) Oxidation-reduction potentials of organic systems, The Williams and Wilkins Co., Baltimore.

26. Johnson, M., Correia, J. J., Yphantis, D. A., and Halvorson, H. (1981) Biophys. J. 36, 575-588.

27. Laue, T. M., Shah, B. D., Ridgeway, T. M., and Pelletier, S. L. (1992) in Analytical ultracentrifugation in biochemistry and polymer science (Harding, S. E., Rowe, A. J., and Horton, J. C., Eds.) pp 90-125, The Royal Society of Chemistry, Cambridge.

28. Holmgren, A. (1984) Methods Enzymol. 107, 295-300.

29. Prongay, A. J., Engelke, D. R., and Williams, C. H., Jr. (1989) J. Biol. Chem. 264, 2656-2664.

30. Poole, L. B., Higuchi, M., Shimada, M., Li Calzi, M., and Kamio, Y. (2000) Free Radical Biol. Med. 28, 108-120.

31. Mulrooney, S. B., and Williams, J., C. H. (1997) Protein Sci. 6, 2188-2195.

32. Lennon, B. W., and Williams, C. H., Jr. (1997) Biochemistry 36, 9464-9477.

33. Reynolds, C. M., and Poole, L. B. (1999) in Flavins and Flavoproteins 1999 (Ghisla, S., Kroneck, P., Macheroux, P., and Sund, H., Eds.) pp 681-684, Agency for Scientific Publications, Berlin.

34. Zanetti, G., and Williams, C. H., Jr. (1967) J. Biol. Chem. $242,5232-5236$.

35. Zanetti, G., Williams, C. H., Jr., and Massey, V. (1968) J. Biol. Chem. 243, 4013-4019.

36. Massey, V., and Palmer, G. (1962) J. Biol. Chem. 237, 23472358.
37. Lennon, B. W., and Williams, C. H., Jr. (1996) Biochemistry $35,4704-4712$.

38. Williams, C. H., Jr. (1976) in The Enzymes (Boyer, P. D., Ed.) pp 89-173, Academic Press, New York.

39. Fisher, C. W., Shet, M. S., Caudle, D. L., Martin-Wixtrom, C. A., and Estabrook, R. W. (1992) Proc. Natl. Acad. Sci. U.S.A. 89, 10817-10821.

40. Shet, M. S., Fisher, C. W., Homans, P. L., and Estabrook, R. W. (1993) Proc. Natl. Acad. Sci. U.S.A. 90, 11748-11752.

41. Sakaki, T., Kominami, S., Takemori, S., Ohkawa, H., Akiyoshi-Shibata, M., and Yabusaki, Y. (1994) Biochemistry 33, 4933-4939.

42. Aliverti, A., and Zanetti, G. (1997) Biochemistry 36, 1477114777.

43. Miura, Y., and Fulco, A. J. (1974) J. Biol. Chem. 249, 18801888.

44. Correll, C. C., Ludwig, M. L., Bruns, C. M., and Karplus, P. A. (1993) Protein Sci. 2, 2112-2133.

45. Suzuki, M., Hayakawa, T., Shaw, J. P., Rekik, M., and Harayama, S. (1991) J. Bacteriol. 173, 1690-1695.

46. Bruns, C. M., and Karplus, P. A. (1995) J. Mol. Biol. 247, $125-145$.

47. Karplus, P. A., Daniels, M. J., and Herriot, J. R. (1991) Science 251, 60-66.

48. Wieles, B., van Noort, J., Drijfhout, J. W., Offringa, R., Holmgren, A., and Ottenhoff, T. H. (1995) J. Biol. Chem. 270, 25604-25606.

49. Wang, P.-F., Marcinkeviciene, J., Williams, C. H., Jr., and Blanchard, J. S. (1998) Biochemistry 37, 18378-18389.

50. Chae, H. Z., Chung, S. J., and Rhee, S. G. (1994) J. Biol. Chem. 269, 27670-27678.

51. Nogoceke, E., Gommel, D. U., Kiess, M., Kalisz, H. M., and Flohe, L. (1997) J. Biol. Chem. 378, 827-836.

BI000826D 